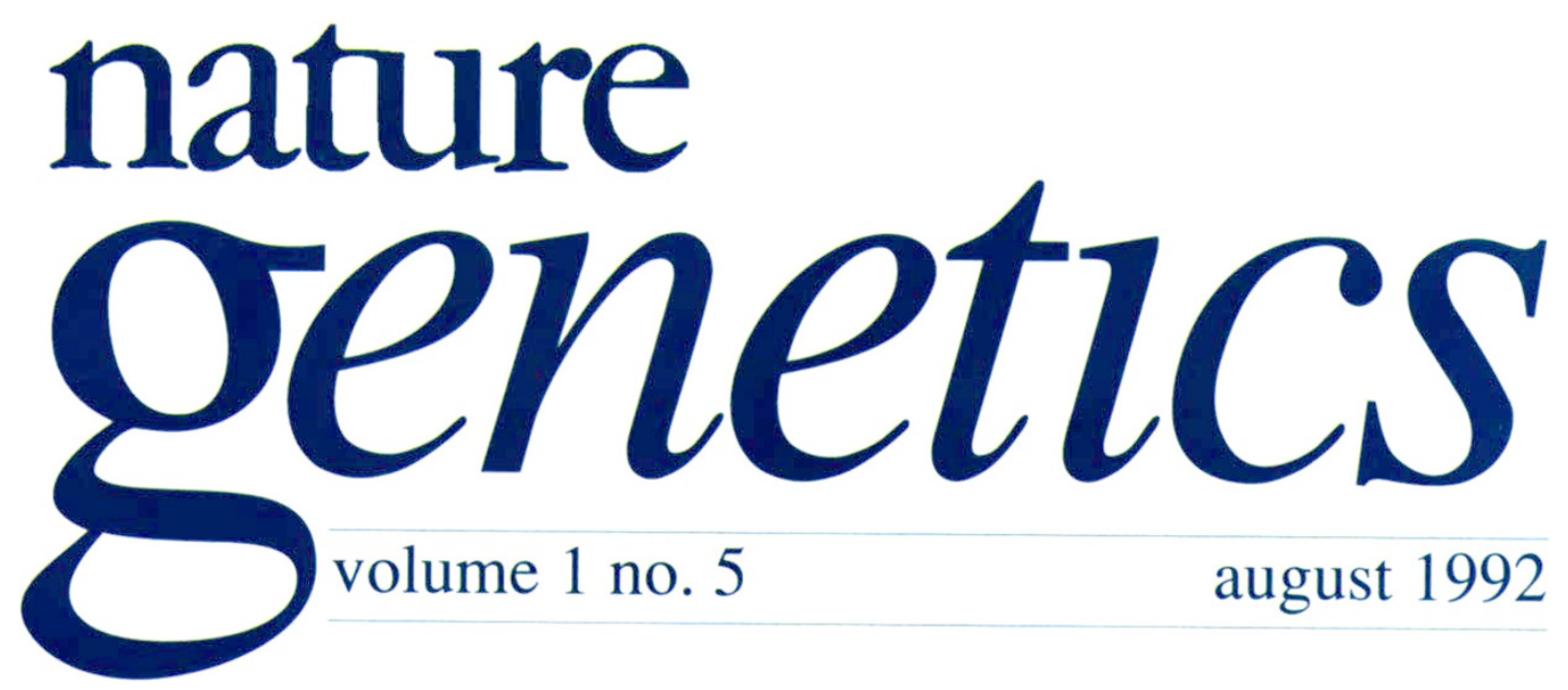

\title{
No way out
}

It is hard to believe, but the three-year anniversary of the discovery of the gene for cystic fibrosis (CF) is upon us. Befitting the very encouraging progress being made in combatting CF, three international conferences will be held during the next few months in Dublin, Paris and Washington D.C., covering every angle of CF research — including diagnosis, management, screening, molecular biology and therapy. Although there is still a great deal more to be learned about the mechanism of action of the cystic fibrosis transmembrane conductance regulator (CFTR) - the defective gene product - the overwhelming evidence points to its principal function as being a cyclic AMPactivated chloride channel. Most certainly it is a channel with an unorthodox structure, quite possibly capable of transporting more than one substrate. Yet, as convincingly demonstrated by John Riordan and colleagues in experiments in which they purified and reconstituted CFTR into lipid bilayers, it is a $\mathrm{Cl}^{-}$channel nonetheless ${ }^{1}$.

Given the rapid pace of discovery, it is not premature to consider in earnest possible therapeutic strategies to relieve patients of their life-threatening lung infections. A number of pharmacological approaches are being tested clinically, and without minimizing the hurdles that remain, gene therapy (for example using adenoviral vectors ${ }^{2}$ ) is a distinct possibility in the future. But as researchers test the efficacy and safety of these various approaches, it would be worth noting the latest observations from Riordan and his coworkers from the Hospital for Sick Children in Toronto, which are presented in this issue. Almost two years after Alan Smith and colleagues at Genzyme Corporation found that many mutant forms of CFTR expressed in vitro are misprocessed, degraded and do not reach the cell surface ${ }^{3}$, Riordan, Norbert, Kartner and colleagues ${ }^{4}$ report analogous findings in vivo in the sweat duct of CF individuals. Their observations may have important consequences for devising strategies aimed at therapuetic intervention.

To understand fully the potential significance of the Toronto group's study, it is worth reviewing the evidence indicating that CFTR is processed incorrectly within the cell. In spite of the caveat that in vitro cell systems may not accurately represent the situation in vivo, Cheng et al.'s results came as a considerable surprise to those in the field. Expressing both wild-type and various mutant constructs of CFTR in COS cells (because the higher protein yields in vitro made expression much easier than in the epithelial cells that normally synthesize CFTR), they found a significant processing defect in numerous CFTR mutants. Wild-type CFTR is glycosylated en route to the cell surface; in contrast, $\Delta F 508$, the most commonly found CFTR mutant, is not processed correctly and failed to reach the cell exterior. Similar abnormalities are found for other mutants (although a minority do reach the cell surface). The startling conclusion is that CF may be caused not by the altered activity of the CFTR protein, but rather because the protein fails to reach its normal position within the cell.

Two groups have since shown that mutant CFTR 
does retain some function in high-expression systems if it reaches the surface. Dalemans and colleagues in France and the NIH found that the vast majority of $\Delta$ F508 CFTR protein expressed in Vero cells failed to reach the surface, but sufficient protein was processed to yield chloride currents, albeit with altered kinetics ${ }^{5}$. Meanwhile, University of Michigan researchers expressed normal and mutant CFTR in Xenopus oocytes, and found that the mutant variants could, under conditions that elevated cAMP levels, produce channel activity ${ }^{6}$.

The notion of a biosynthetic block as the cause of CF is surprising, but not without precedent. Indeed, the list of human diseases thought to be caused, at least in part, by a similar intracellular processing defect, is almost in double figures and is likely to grow considerably ${ }^{7}$. The most notable examples are some classes of LDL receptor variants in familial hypercholesterolaemia.

Nevertheless, the debate about the significance of CFTR processing in vitro must be transfered to a more physiological setting, and that is what has now been achieved ${ }^{4}$. The new study focuses on sweat glands, obtained by skin biopsies of healthy and affected individuals. This has the advantage of examining a tissue affected by $\mathrm{CF}$ (patients were first diagnosed in the ' 50 s by virtue of having elevated levels of salt in their sweat) but lacking any of the secondary manifestations, such as inflammation.

Kartner and coworkers used a panel of monoclonal antibodies that recognize different domains of CFTR (and detect normal and $\Delta$ F508 proteins equally well) to examine the location of the protein in normal and affected sweat glands (in addition to other tissues). In normal biopsies, CFTR was especially prominent in the apical membrane of the reabsorptive sweat duct. In contrast, samples from $\Delta F 508$ homozygotes showed no apical membrane staining, while those of heterozygous carriers showed only quantitative differences compared to normal.

The parallel with the in vitro findings in COS cells is sustained in studies of a CF patient containing a Gly to Asp substitution at residue 551 (G551D) in one allele. In COS cells, G551D CFTR was processed correctly, and in contrast to $\triangle F 508$ CFTR, this protein is detected on the apical membrane in vivo. Sweat glands from two other patients possessing rare CFTR alleles who are mildly affected with CF also revealed apical localization of CFTR. Finally, a number of CF homozygote sweat glands showed evidence for dense, 'granular' staining within some cells, including the sweat coil, again indicative of a processing defect.

Taken together, these findings strongly suggest that, in severely affected CF patients, the majority of the CFTR cannot reach the surface, thereby preventing it from performing anywhere near full capacity. It remains to be seen to what extent the findings in CF sweat glands are mimicked in airway epithelia - the most clinically important tissue. Although $\triangle F 508$ CFTR has been claimed to localize correctly in airway cells, Riordan and coworkers dismiss the available evidence in characteristically forthright manner. (Indeed, Riordan seldom balks at an opportunity to challenge controversial results in print, and has on occasion branded published conclusions as 'simplistic' or 'without credence'.)

Kartner, Riordan and colleagues conclude that "potential pharmacological solutions to CF may first need to solve the problem of redirecting the processing of $\Delta$ F508 CFTR" to enable the protein to reach the plasma membrane - essentially the prediction of Smith and colleagues in 1990. However, finding an agent that might somehow guide CFTR (or any protein for that matter) through the endoplasmic reticulum to its correct location, without compromising protein biosynthesis in general, will be an arduous task. Concern has also been voiced that the amount of normal CFTR introduced into epithelial cells by protein or gene replacement strategies might affect the clinical outcome. But two encouraging studies to be published soon in Nature Genetics suggest that this may not be a problem. J. Whitsett, from the Children's Hospital, Cincinnati, and collaborators have created healthy transgenic mice expressing high levels of CFTR in respiratory epithelial cells ${ }^{8}$. And L. Johnson and colleagues from the University of North Carolina find that only a relatively small number of 'corrected' CF cells are necessary to restore normal chloride transport properties in vitro ${ }^{9}$. Perhaps the forthcoming round of CF conferences will yield further advances on these pressing questions.

\footnotetext{
1. Bear, C.E. et al. Cell 64, 681-691 (1992).

2. Rosenfeld, M.A. et al. Cell 68, 143-155 (1992).

3. Cheng, S.H. et al. Cell 63, 827-834 (1990).

4. Kartner, N. et al. Nature Genet. 1, 321-327 (1992).

5. Dalemans, W. et al. Nature 354, 526-528 (1991).

6. Drumm, M.L. et al. Science 254, 1797-1799 (1991).

7. Amara, J.F. et al. Trends Cell Biol. 2, 145-149 (1992).

8. Whitsett, J. et al. Nature Genet. (in the press).

9. Johnson, L.G. et al. Nature Genet. (in the press).
} 